\title{
CARACTÉrISATION DES MIELS DE L'OCÉAN INDIEN PAR SPECTROMÉTRIE PROCHE INFRAROUGE : ÉTUDE DE FAISABILITÉ
}

\author{
ChaRACTERIZATION OF HONEYS FROM THE INDIAN OCEAN \\ USING NEAR INFRARED SPECTROMETRY: FEASIBILITY STUDY
}

\author{
CARACTERIZACiÓN DE MIELES PROCEDENTES DEl OCÉANO INDICO \\ POR ESPECTROSCOPÍA DE INFRARROJO CERCANO: ESTUDIO DE VIABILIDAD
}

\author{
S. Nabeneza ${ }^{1 *}$ V. Porphyre ${ }^{1}$ F. Davrieux ${ }^{2}$
}

Mots-clés : Miel - Spectroscopie proche infrarouge -

Provenance - Adultération des aliments - Océan Indien.

Keywords: Honey - Near infrared spectroscopy -

Provenance - Food adulteration - Indian Ocean.

Palabras clave: Miel - Espectroscopía de infrarrojo cercano Procedencia - Adulteración de alimentos - Océano Indico

e miel est traditionnellement consommé dans les îles de

Ll'océan Indien et utilisé également pour ses propriétés cosmétiques et thérapeutiques. Ce produit, issu du nectar de fleurs ou de miellat d'insectes, est collecté et transformé par les abeilles produisant ainsi un miel unique caractéristique de la flore de chaque île. A l'issue de la récolte, I'apiculteur doit normalement veiller à ce que le miel soit conforme et respecte des caractéristiques physico-chimiques spécifiques selon les recommandations du Codex alimentarius. De plus, il doit indiquer des informations, comme l'origine botanique et géographique, sur chaque pot de miel vendu. En pratique il est toutefois très difficile de vérifier l'exactitude de ces informations, d'autant qu'elles sont mises en avant comme argument de vente.

La spectrométrie dans le proche infrarouge (SPIR), basée sur les propriétés physiques d'absorption de la lumière par les composés organiques, peut être un outil de contrôle et de traçabilité des miels mis sur le marché $(1,3)$. L'objectif principal de cette étude a été d'évaluer la faisabilité d'un contrôle qualité (authentification de l'origine botanique et/ou géographique et détection d'adultération) des miels commerciaux produits dans I'océan Indien. Les perspectives de cette méthode de laboratoire sont importantes dans le cadre du développement des filières apicoles, notamment lorsqu'elles sont orientées vers les marchés d'exportation et vers la promotion de leur qualité auprès de consommateurs désireux de profiter de produits d'excellence et fortement attachés à des territoires insulaires uniques.

1. Cirad, UMR Selmet, station de Ligne-Paradis, 7 chemin de l'IRAT, 97410 Saint-Pierre, Réunion, France.

2. Cirad, UMR QualiSud, station de Ligne-Paradis, 7 chemin de l'IRAT, 97410 Saint-Pierre, Réunion, France.

* Auteur pour la correspondance

E-mail : serge.nabeneza@cirad.fr
Au total, 625 miels des pays de l'océan Indien (tableau I) ayant diverses origines botaniques (tableau II) ont été mesurés sans ajustement à $30{ }^{\circ} \mathrm{C}$ et après ajustement à $70^{\circ}$ Brix avec de l'eau distillée (3). La mesure a été réalisée en réflexion diffuse à l'aide d'une cellule ronde avec un fond réflecteur doré (trajet optique $0,1 \mathrm{~mm}$ ) sur un spectromètre FOSS NIRSystem 5000 (1 100-2 500 nm, 2 nm). En parallèle, les mesures du degré Brix, de l'humidité et de la conductivité des miels bruts ont été réalisées au laboratoire. Après l'analyse en composantes principales (ACP) de la base spectrale des 625 miels et le calcul des distances $\mathrm{H}$ de Mahalanobis, 83 miels ont été identifiés comme spectralement atypiques et retirés du jeu de données (tableau I). Sur la base des distances spectrales ( $\mathrm{H}$, Mahalanobis), 64 miels représentatifs des 542 miels retenus ont été sélectionnés, ajustés à $70^{\circ}$ Brix, puis adultérés avec du sirop de sucre de canne du commerce (Mascarin®) à 25 et à 10 p. 100 par pesée.

\section{Tableau I}

Origine géographique des miels de I'océan Indien

\begin{tabular}{lrrr}
$\begin{array}{l}\text { Origine } \\
\text { géographique }\end{array}$ & Madagascar & Réunion & Rodrigues \\
\hline Nombre total & 262 & 231 & 132 \\
Echantillons atypiques & 33 & 34 & 16 \\
Echantillons retenus & 229 & 197 & 116
\end{tabular}

Tableau II

Origine botanique des miels de I'océan Indien

\begin{tabular}{lrrr}
$\begin{array}{l}\text { Origine } \\
\text { botanique }\end{array}$ & Madagascar & Réunion & Rodrigues \\
\hline Litchi & 52 & 45 & 0 \\
Eucalyptus & 42 & 0 & 71 \\
Mille fleurs & 47 & 31 & 19 \\
Baie rose & 0 & 63 & 0 \\
Niaouli & 25 & 0 & 0
\end{tabular}


Les modèles prédictifs des teneurs en humidité, Brix et conductivité ont été établis en utilisant la régression modified partial least square (M-PLS) du logiciel WinISI 3 (Infrasoft, Port Mathilda, PA, Etats-Unis). Les modèles PLS ont été réalisés sur la base des dérivées premières des spectres normalisés (SNV) et corrigés pour la ligne de base (Detrend). Pour expliquer les critères géographiques et botaniques, nous avons utilisé une méthode non supervisée de discrimination (Cluster Analysis, Unscrumbler 10.3, CAMO, Oslo, Norvège), qui constitue des groupements naturels, sur la base des distances spectrales. Les spectres des miels non adultérés ont servi de base pour définir un espace multidimensionnel (ACP centrée, non réduite sur dérivée seconde des spectres bruts). La projection des spectres (dérivée seconde des spectres bruts) des miels adultérés sur cet espace a permis d'identifier un plan factoriel (CP1-CP7) expliquant 65 p. 100 de la variance totale, sur lequel les miels adultérés étaient nettement séparés des miels non adultérés. Les modèles d'étalonnage obtenus par régression PLS pour le degré Brix, I'humidité et la conductivité ont été performants avec des $\mathrm{R}^{2}$ de I'ordre de 0,92 et des erreurs de validation croisée acceptables (tableau III) (figure 1).

Les essais de groupement des individus sur la base de leur spectre (recherche de clusters), de même que I'utilisation d'autres méthodes mathématiques, comme l'analyse discriminante linéaire (LDA) et la classification support vecteur machine (SVM) à partir des origines géographiques et botaniques n'ont pas donné de résultats satisfaisants (tableau IV). Cette impossibilité de discriminer les miels de l'océan Indien serait peut-être due à une identification botanique imprécise des miels. Sans analyses polliniques des échantillons de miels, les origines botaniques ne peuvent être certifiées, en particulier les miels dits monofloraux. Plusieurs hypothèses peuvent expliquer l'impossibilité de discriminer les miels de l'océan Indien en fonction de leur origine géographique et/ou botanique. Parmi elles, les différences chimiques caractéristiques de l'origine géographique et/ou botanique correspondent à des classes de composés (aromatiques par exemple) qui n'impactent pas suffisamment les empreintes spectrales, ne permettant pas de trouver des facteurs discriminants. La projection des miels adultérés à 25 p. 100 (figure 2), sur la base de miels non adultérés montre une séparation nette des populations. En revanche, pour un niveau d'adultération à 10 p. 100, la séparation n'est pas évidente (figure 3).

Les résultats obtenus montrent que la spectrométrie dans le proche infrarouge peut être utilisée pour prédire certains paramètres physico-chimiques des miels. Ces premiers résultats montrent que la constitution d'une base spectrale de miels adultérés à différents niveaux au laboratoire, associée à des méthodes de régression ou de discrimination devrait permettre I'identification de miels non-conformes, comme montré précédemment par Rios-Corripio et coll. (2).

Ces résultats ne permettent pas, dans l'état actuel, de différencier l'origine géographique et botanique des miels collectés dans le

\section{Tableau III}

Paramètres statistiques des modèles d'étalonnage des miels de l'océan Indien

\begin{tabular}{lccccccc} 
Constituant & N & Moyenne & Min & Max & SEC & R $^{2}$ & SECV \\
\hline Humidité $(\%)$ & 494 & 19,4 & 14 & 24,7 & 0,53 & 0,91 & 0,54 \\
${ }^{\circ B}$ Brix & 415 & 79,6 & 74,4 & 84,7 & 0,49 & 0,92 & 0,51 \\
Conductivité $(\mu S)$ & 328 & 1003 & 201 & 2682 & 154 & 0,92 & 211
\end{tabular}

Moyenne, Min, Max : statistiques de la population d'étalonnage ; SEC : erreur standard de calibration ; $\mathrm{R}^{2}$ : coefficient de corrélation du modèle d'étalonnage ; SECV : erreur standard de validation croisée

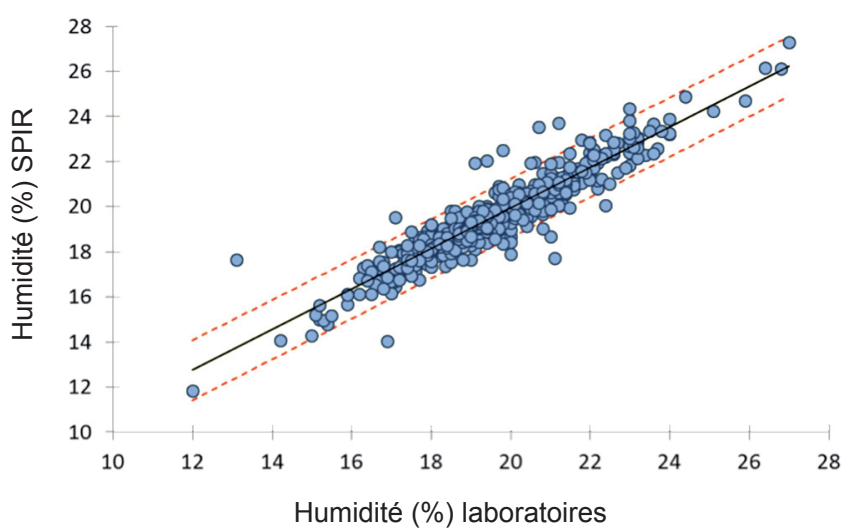

Figure 1 : corrélation entre les valeurs d'humidité au laboratoire et de la spectrométrie dans le proche infrarouge (SPIR) des miels de I'océan Indien.

\section{Tableau IV}

Répartition des miels de l'océan Indien dans les groupes après discrimination

\begin{tabular}{lrcc} 
& \multicolumn{3}{c}{ Origine géographique réelle } \\
\cline { 2 - 4 } $\begin{array}{l}\text { Groupement } \\
\text { (cluster) }\end{array}$ & Madagascar & Réunion & Rodrigues \\
\hline Madagascar & 107 & 79 & \\
Réunion & 41 & 73 & 51 \\
Rodrigues & 81 & 45 & 20 \\
Nb. total de miels & 229 & 197 & 116
\end{tabular}




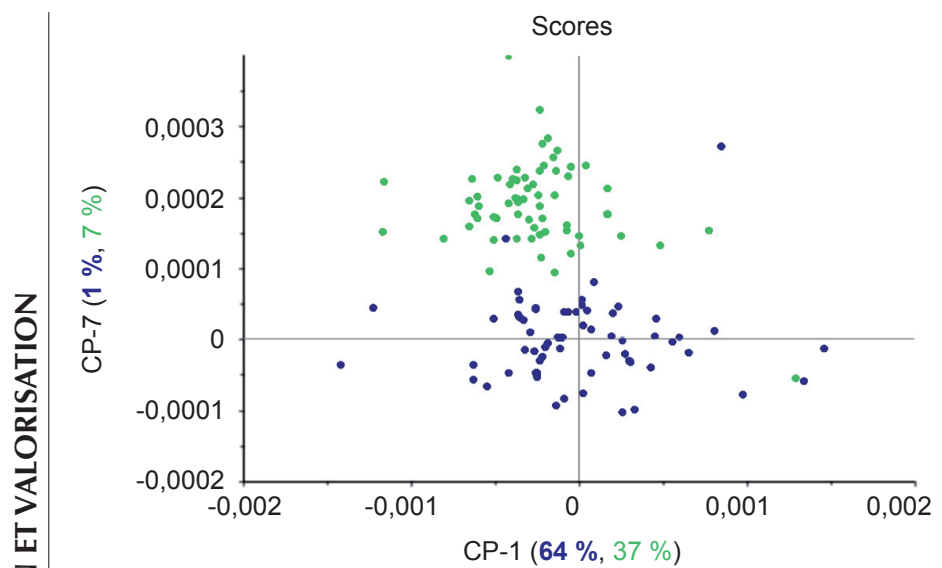

Figure 2 : projection de 64 miels de l'océan Indien adultérés à 25 p. 100 sur le plan factoriel calculé par analyse des composantes principales $(A C P)$ à partir des 64 miels non adultérés représentatifs des 542 miels retenus.

commerce. La limite de détection des miels adultérés par du sucre de canne devra être déterminée. Elle se trouve entre 10 et 25 p. 100. Les fraudeurs adultérant fréquemment les miels avec du glucose, il serait important de poursuivre les essais avec ce produit. II est donc possible de détecter, par la technique SPIR, des altérations importantes du miel par ajout de sucre de canne.

\section{BIBLIOGRAPHIE}

1. COZZOlinO D., CORBEllA E., SMYTH H.E., 2011. Quality control of honey using infrared spectroscopy: A Review. Appl. Spectrosc. Rev., 46: 523-538. DOI:10.1080/05704928.2011.587857

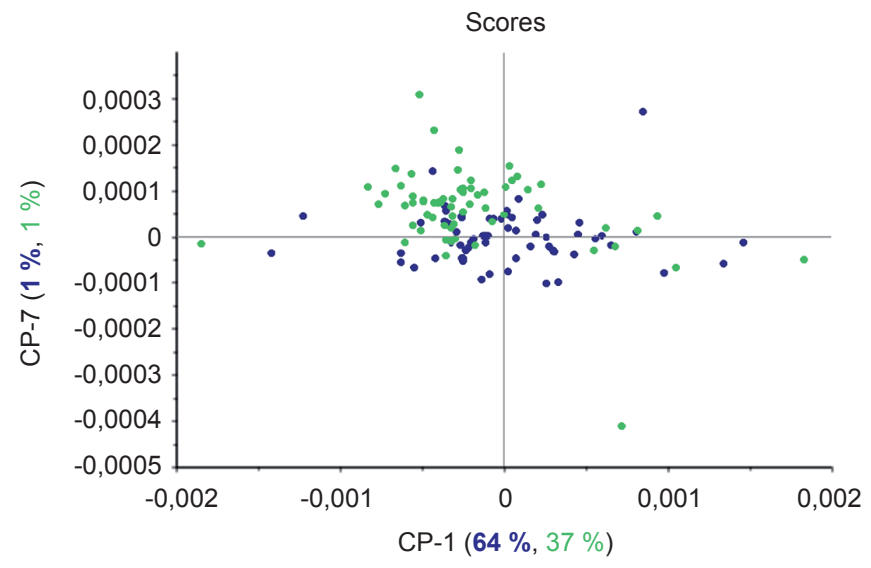

Figure 3 : projection de 64 miels de l'océan Indien adultérés à 10 p. 100 sur le plan factoriel calculé par analyse des composantes principales $(A C P)$ à partir des 64 miels non adultérés représentatifs des 542 miels retenus.

2. RIOS-CORRIPIO M.A., ROJAS-LOPEZ M., DELGADO-MACUIL R., 2012. Analysis of adulteration in honey with standard sugar solutions and syrups using attenuated total reflectance-Fourier transform infrared spectroscopy and multivariate methods. CYTA - J. Food, 10: 119-122. DOI: 10.1080/19476337.2011.596576

3. WOODCOCK T., DOWNEY G., O'DONNELL C.P., 2009. Near infrared spectral fingerprinting for confirmation of claimed PDO provenance of honey. Food Chem. 114: 742-746. DOI:10.1016/j. foodchem.2008.10.034

Accepted 30 April 2015; Online publication June 2015 